\title{
Mobile Game for the Elderly: Bundled Bingo Game
}

\author{
Yi Jia Chin, Woan Ning Lim, Chien Sing Lee \\ Department of Computing and Information Systems \\ School of Science and Technology \\ Sunway University \\ Bandar Sunway, Malaysia. \\ ( woanningl@sunway.edu.my, chiensingl@sunway.edu.my )
}

\begin{abstract}
The aim of this project is to develop a customized Bingo game based on the user interface guidelines that suit the elderly users. This project targeted to improve the cognitive ability of the elderly with the specifically designed gaming features. In this research, qualitative data will be used to analyze the confidence and acceptance level of the elderly on the mobile digital games, as well as to understand how the elderly interacts with current technologies (mobile devices) and the benefit they can gain from the mobile games. User testing, survey, and result analysis have been done with a group of elderly members from D'Happy club in Petaling Jaya, Malaysia. The results of this project contribute to the development of an elderly friendly mobile game to motivate the elderly's engagement in mobile gaming, with the will that it could help to delay and decrease the risk of the elderly in developing the Alzheimer's disease.
\end{abstract}

Keywords - bingo; mobile game; elderly; cognitive

\section{INTRODUCTION}

The increases in the elderly population nowadays has led to the development of technologies that is beneficial to the elderly users. Smart phone is important for the elderly as they can use it to communicate with their families and making friends, and as a tool to enhance the independent and safety. Most of the elderly realized that they have to adapt to the new technologies by using smart phone that is beneficial for their daily activities [1]. In addition, smart phone device can act as a companion to reduce the feeling of loneliness, and indirectly help to enhance the elderly's cognitive condition where the brain can be stimulated and memory can be strengthened when they play the games. Some researchers are into investigating the possibility of mobile gaming in prevention of Alzheimer's disease [2].

With the advancement of the 21 st century, smart phone developers start to realize the increasing in usage of elderly technology user and hence, they start to develop more related and appropriate technologies that suit the elderly. The elderly can gain benefits from using technology such as being more productive and connected to other people and services as well as using technology as a tool for improving their health [3]. Unfortunately, most of the current technologies which are available are not suitable for older users as the elderly find that it is difficult to understand how to perform the task due to aging symptoms and bad perception about smart phone. Besides that, their memories may not able to work as well as

The research was funded by the Malaysian Ministry of Education's Fundamental Research Grant Scheme FRGS/1/2016/ICT04/SYUC/01/1 (Aug 2016-Jul 2018) the young generation and they will need considerable time in order to master the controls of the smartphone [1].

According to Duh [4], elderly people are increasingly adopting technologies such as computers, Internet and electronic devices. As such, mobile devices are rapidly becoming personal items for them. Therefore, including games to mobile devices that are suitable to them will enable them to play games that can help to improve their healthcare and cognitive reflexes. However, in most cases mobile devices are kept for emergency calling or as memory aids, and yet few games are currently developed for the elderly users. With the increasing number of elderly users using mobile devices, the functionalities of the smart phone devices should focus on their knowledge level and their misgiving. Misgiving includes characteristics such as motivations, low frustration level and having to overcome previous, negative experiences must be taken care in developing mobile games for elderly [5].

The current demography of the elderly do not reach the same level technology as the young generation. The fact is that many elderly retired without the need of using technologies such as computers or internet during their working time [6]. Mobile games that targets young generation result in the increased access barrier to the gaming for elderly, making the elderly do not want to adapt themselves to the same level technology as young generation. As such, this may eventually cause great impact to elderly's desire and confidence level in playing mobile games [7]. Besides that, elderly have negative perception towards multi-player games. This could be arise due to the fear of failure and unwillingness to be tied to specific times when others would be available to play with [8]. In addition, elderly seem to hold concerns about the negative effects of the mobile gaming activity on gamers such as violent genre games. It is important to take note that 'speed' and 'concentration' are not considered important elements to elderly. Instead, they would like to prefer more 'relaxation', 'fun' and 'amusement' gaming system [9].

This project aims to build a customizable Bingo mobile game that suit to the elderly needs. Bingo is a board game which contains a grid of at least nine squares. Each square is represented by random numbers. Some customization Bingo game can be represented by words or pictures. The bingo game is played by having a person randomly draw from a pool of numbers, and the player will cross the number on his/her Bingo card when the number is called. Whoever that able to cross a complete diagonal, horizontal or vertical row will win the 
game. The Bingo game developed in this project is a customizable game which allow user to play up to three Bingo board at the same time, which encourage player to think and plan their strategy, hence help in enhancing the users' cognitive reflexes. The game was also designed to bundle with a response numbers game which allow the user to train their reflex action and constantly improve their response. The game was designed using proper interface that is easy for interaction with elderly as well as with good visual effect. The aim of this mobile gam is to allow the elderly users to enjoy the game and be entertained at the same time enhance their cognitive responses. Rautenberg [10] states that entertainment technology has positive effects on human behavior such as: learning ability, positive social interaction and healthcare.

\section{INTERFACE DESIGN GUIDELINE FOR ELDERLY}

Generally, it is known that elderly people use their mobile devices for restricted purpose. The reasons of why elderly people use phones for limited purpose are due to the hard-tosee display, hard-to-learn procedures and hard-to-press buttons as the latest mobile devices are designed for young generation. Young people could easily master on how to operate the functionality of the mobile devices. On the other hand, the elderly find that it is hard for them to master in using mobile devices [11].

Interface is an important factor in designing the game as player direct interact with the smartphone device through interface. The ageing process changes both biological and physical levels in human body. These changes could limit a person to perform certain daily activities. This can be seen when the elderly users are interacting with the smart phone devices where the devices have yet fully accommodated their limitations. Therefore, the game must be designed carefully to meet elderly peoples' requirements considering their health condition and limitations. This can be done by adjusting the font size, suitable naming conventions and icons for several functionalities that are desirable for elderly [12]. In addition to adjusting the font size, naming conventions and icons, sensitivity controls and simplifying task such as inputting text or touch interactive buttons can help to prevent confusion in playing games and reduce elderly users' frustration levels [13]. According to Vasconcelos [12], direct devices are easier to use as it requires lesser coordination due to little hand-eye coordination and minimal spatial demands as compare with indirect devices such as controllers which are more precise and preferred by users with experiences for more meaningful gaming experience. Due to the characteristics of direct devices, direct input mobile devices are more suitable for senior users.

Visual information and 'useless' information should be removed when they are playing games. For example, advertisement, colored-text and visual animation can cause confusion and they are unable to catch the flow of the game. They will have trouble in recognizing and selecting the functions amongst other information showed on the screen when playing mobile games. Simple interface design is efficient enough for elderly users to prevent them from suffering frustration and confusion in using mobile devices
[14]. In terms of in-game graphic, the contrast and color settings should be adjustable in order to meet individual needs [15].

When designing the clickable target size, it is recommended to use large targets as elderly can make errors easily such as choosing the wrong target if the target is small. Therefore, making the targets big could reduce the error of tapping wrong targets [11].

Other than that, there are some important elements to be considered when designing interface for elderly users. These important features are instant feedback, cognitive challenge design, customization of the game, interaction preference, and motor features.

\section{A. Instant Feedback}

It is recommended to implement the games that can provide instant feedback. The elderly users are aware of the impact of the actions for they have chosen through the feedback mechanism. For example, when playing game, the actions made by users will be responded with feedback either if they have succeeded the stage through the actions or failed with detail explanations of why they failed. With this instant feedback information, the elderly users are able to improve their reflexes such as brain responses while remaining motivated by their actions' outcome [12].

Other than that, the game could be implemented that provides multiple modes feedback other than a single communication channel that combines various input and output modalities. For instance, feedbacks such as seeing, hearing and touching can be useful for elderly as it gives the user a response whilst interacting with the game. The reason of introducing multiple modes feedback is to increase the usability by covering the weakness of one form of feedback by another [14].

Providing visual feedback to show where the users touch the screen or when they missed the interactive target can be beneficial as they are able to track their movement. The indicators of the feedback should be large and medium duration as this ensure that the elderly users will be able to notice the alert or feedback or changes in look-and-feel. More importantly, the feedback must be understandable and readable with simple language instead of using symbolic representation. The use of symbolic representation as feedback form can lead the elderly to have problem in understanding the meaning of the symbolic representation [11].

\section{B. Cognitive Challenge Design}

According to Oxford Dictionaries, cognition is defined as: "The mental action or process of acquiring knowledge and understanding through thought, experience, and the senses."

Age-related changes in cognitive can affect the requirements of the game interface design. The elderly will encounter the problems such as the decline in cognitive function process which include inability to keep focus, degradation in working memory, difficulties in solving a problem and reasoning as well as memory encode-decode process. Apparently, remembering information from one screen to another screen could be difficult due to the 
degradation in memory. The elderly users tend to be forgettable and they are not able to remember things from screen to screen. With that in mind, the main focus on the interface has to be simple and intuitive, giving adequate affordance and overview. This can be done by having the elderly users to just interact with everything in one screen instead of multiple screen choices. This helps keep the load on memory and cognitive processing to a minimum level stage [16].

The mobile game implementation should be neither simple nor complex so that elderly should feel comfortable and confident while playing the game. Therefore, they can enjoy playing the game because of the challenging and simple usability features provided by the game and they will get motivated in continue playing the game [17]. Hence, we could relate this with feedback features as it plays an important role to make sure that the elderly could enjoy playing the game with the game providing positive feedback to them [16].

As conclusion, the game should be designed in meaningful way that are perceivable by elderly and enabling them to require some thinking skills in playing the game. Although the game is designed to be user-friendly but if it does not provide the fun factors to the elderly users, they might get uninterested in playing the game. And, to note that counterstrike with adjustable font size may not be the "killer application" for elderly users." [16].

\section{Customization}

How people viewed others can be greatly affected by the "favorable vs unfavorable" or "positive versus negative" factors [18]. The statement above has strong relationship when it comes to the game customization as well. This can be seen from the perspective such as different users have different experiences. The users tend to choose the games that can create enjoyable time to them. Therefore, the game should be customizable and flexible by meeting individuals' needs by introducing variety familiar pictures, videos or sounds that can improve users' overall experience. Moreover, to cover a wide range of users so that even elderly can have fun playing the games without any problem, the game user interface in game design must be customizable in terms of its font's size or the icons that can be adjusted to meet individual needs [12].

As mentioned earlier, the elderly people should be able to choose the difficulty of the game as well as the adjustment of game speed and sensitivity control. The game difficulty should be adjustable so that it can meet individual's experience. This is important because if the game is too hard to clear, user will be unmotivated and eventually stop playing that particular game. Hence, the game should not be very hard for elderly use as due to their cognitive function degradation, they may not able to clear certain stages and they cannot make any progress in the game [12].

Besides that, time management is an important element to be included when developing the mobile game. This is because the elderly are often on strict time table with other activities that are planned in advance. Therefore, time management ensures elderly users to be able to balance the time of playing games and their planned activities.
Language support feature is considered another crucial element criterion in developing mobile games. This could be due to not all of the elderly people are English educated. The difficulty in interpreting and understanding the language can discourage the elderly to stop playing particular games as they do not have choices to choose other languages that are favorable to them. The difficulty in understanding the language will prevent them from proceeding another stages provided the current stage needs some written hints to unlock the next stage. With language support features, language barrier which could cause hindrance to meaningful play could be solved by including automated translation tools, or an option to choose preferably language in-game play [19].

In short, each user has different view on determining whether the game is acceptable for own personal needs and hence, it is very important that the game should be flexible and customizable to meet individual requirements as well as accommodating a wide range of users with the feasibility in the mobile game design. It is a good practice that the game interface should not be adjusted that will exceed the beyond boundaries for playability of a game. For example, 200 font size on a portable game device do not necessarily increase its readability. By allowing the users to manually control font size, color and contrast setting, it can further increase the readability chance [16].

\section{Motor Features}

"Motor Skills is defined as the ability to perform complex muscle-and-nerve acts that produce movement" [20].

Motor impairments have different impact on user experience. Elderly people is facing the degradation in cognitive response. They may experience in the changes in motor skills such as slow brain processing power, slow response time, coordination, and loss in flexibility. Thus, any moveable interface element should be avoided when developing a mobile game for elderly users because the elderly users could not able to catch the pace of the flow of ingameplay [16].

The mobile game should focus on physical therapy and different adaption of motor skills. As such, the elderly users would gain benefit from cognitive response, physical and social activity [15].

\section{E. Interaction Design}

Interface provides direct interaction with elderly users. It is common that users with gaming experience are generally familiar with the interface elements and thereby require only little instruction and support in order to master the controls in playing the game. In contrast, elderly users lack of gaming experience [15]. They are not able to adapt themselves to the game because of insufficient guidelines that are provided by the game. They need time to master the game control in order to play the game. The insufficient guidelines on how to play a game can lead to unpleasant playing experience and users will get unmotivated. As such, it is vital that the game interface design should be straightforward and basic in addition providing helpful feedback to help avoid frustration as well as introducing enjoyable player experience. 
The interaction mechanisms of mobile games that are suitable for elderly users can be implemented in following ways. Firstly, the rules and the goals that define the nature of the games must be clear and understandable. Secondly, ingame play should adapt to players' real life and schedule time. Following that, the game should make sure that the players reach a certain proficiency. Lastly, the game concepts include the real life theme and familiar concepts that the players will feel familiar with the game. Ultimately, the game design should have clear goal so that players could understand the nature of the games that they are playing [12].

Another feature to be used in interaction design is by creating imaginary vicarious (secondary) player. According to Oxford Dictionaries, Vicarious is defined as "Experienced in the imagination through the feelings or actions of another person". This can be explained by having secondary helper to give explanation to the users when playing games. For example, providing hints at the corners of the screens, or searching through internet to obtain clues helps the players to advance the stages if they are not to making any progress [12].

\section{BINGO MOBILE APPLICATION}

The Bingo mobile application that was developepd in this project consists of two types of game, which are Start Go and Go Response. The user can choose the game options from the menu screen as shown in Fig. 1.

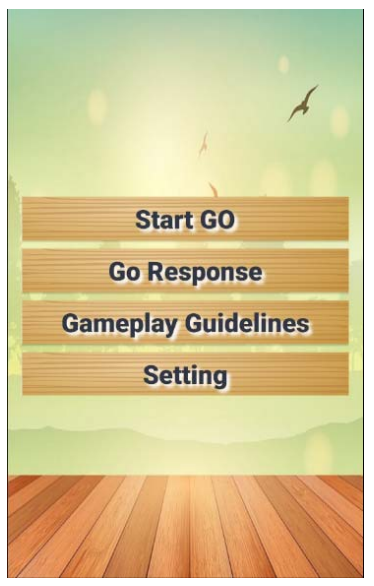

Fig. 1. The main menu of the Bingo game.

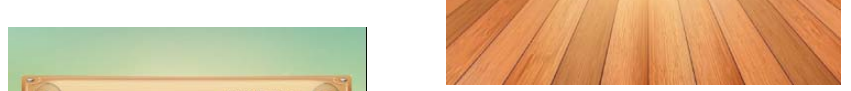

Fig. 3. The Go Response game.

of the screen. User can identify the most strategy board with the two numbers located at the winning row, in order to win the game in the shortest time. This customize Bingo game is different from the traditional Bingo game with only one board active at one time, where no cognitive load is required as the user just need to mark the number when it is called.

The game is designed with the instant feedback feature; when a number is draw, there will be sound spell out the number in the language selected by the user. And, upon any wrong marking by the user, a warning sound will be prompted. And, upon a success marking of a number, the correspondence square will be masked out in yellow. The sounds and colors are used to improve the feedback to the elderly to ease their playing of the game.

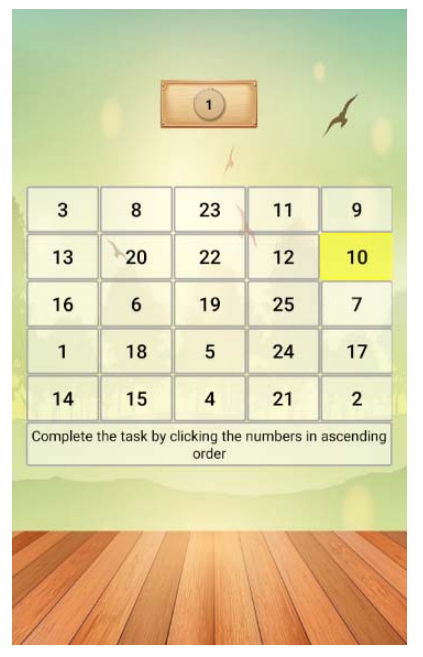

The second option is the Go Response game; the user will be redirected to the responsive game screen which the user will be prompted to choose the total numbers to play (user can choose between 25 or 50 numbers). After choosing the choice of total numbers, the responsive game will automatically start as shown in Fig. 3.

This is a simple game where the user just need to click on all the numbers in ascending order to win the game. This game aims to help the user to improve the response time and cognitive reflex. When the user won the game, the total time taken will be displayed and recorded into the cloud database so that in the long run, the data can be used to analyze the users' improvements in the response behaviors.

The application also equipped with a simple one-page guideline on how to play the two games. This will help to prevent confusion when the user is playing the game. There is also a settings screen which allow the user to configure the language, volume and brightness of the application, as shown in Fig. 4.

\section{RESULTS}

Survey questionnaire consists of 13 questions based on the ARCS (Attention, Relevance, Confidence, Satisfaction) with Cognitive model [21] was used to analyze the cognitive, strategize in choosing which board to mark the numbers with the hints of next coming number shown at the top right corner

If user choose the Start Go game, the user will be redirected the prompt the the number of boards to play (user can choose from automatically as shown in Fig. 2.

User can navigate between the boards by swiping on the screen or click on the left/right arrow button. The implementation of the multiple boards enables the user to 
confidence and acceptance level of the elderly on playing the mobile game. Questions 1-2 evaluate the cognitive load of mental effort and perceived difficulty. Questions 3-6 assess the attention, questions 7-9 evaluate the relevance and questions 10-12 assess the confidence. The last question is to gauge the satisfaction of the overall experience. The questions are as per listed in TABLE I. This survey is run by voluntarily basis, and has obtained $43 \%$ response rate with 10 out of 23 elderly took part in the survey. The low response rate is due to some of the elderly have an older model smart phone or device that is not compatible. Besides that, some of the elderly were reluctant to try and not interested in the mobile technology.

\section{TABLE I. SURVEY QUESTIONS}

\begin{tabular}{ll}
\hline Cognitive \\
\hline Q1 & I use a lot of mental effort to finish [the task]. \\
\hline Please indicate the degree of difficulty level in completing [the \\
task].
\end{tabular}

Seven-point Likert scale is used in the questions, with 1 as 'Strongly Negative' and 7 as 'Strongly Positive'. A summary of the findings, the means, standard deviations and skewness are listed in TABLE II and Fig. 5.

TABLE II. SURVEY RESULTS

\begin{tabular}{lrrrrrrrrrrrrr}
\hline & Q1 & Q2 & Q3 & Q4 & Q5 & Q6 & Q7 & Q8 & Q9 & Q10 & Q11 & Q12 & Q13 \\
\hline $\mathbf{N}$ & 10 & 10 & 10 & 10 & 10 & 10 & 10 & 10 & 10 & 10 & 10 & 10 & 10 \\
Mean & 4.1 & 3.9 & 4.5 & 4.9 & 5.2 & 3.6 & 4.2 & 3.9 & 3.8 & 4.1 & 4.1 & 3.9 & 4.2 \\
Std. Deviation & 1.3 & 1.3 & 1.4 & 1.4 & 1.3 & 1.4 & 1.0 & 0.9 & 1.1 & 1.5 & 1.7 & 0.9 & 1.5 \\
Skewness & -0.2 & 0.2 & -0.6 & -0.1 & -0.1 & -0.5 & 0.3 & 0.2 & -0.7 & -0.2 & -0.2 & 0.2 & -0.9 \\
\hline
\end{tabular}

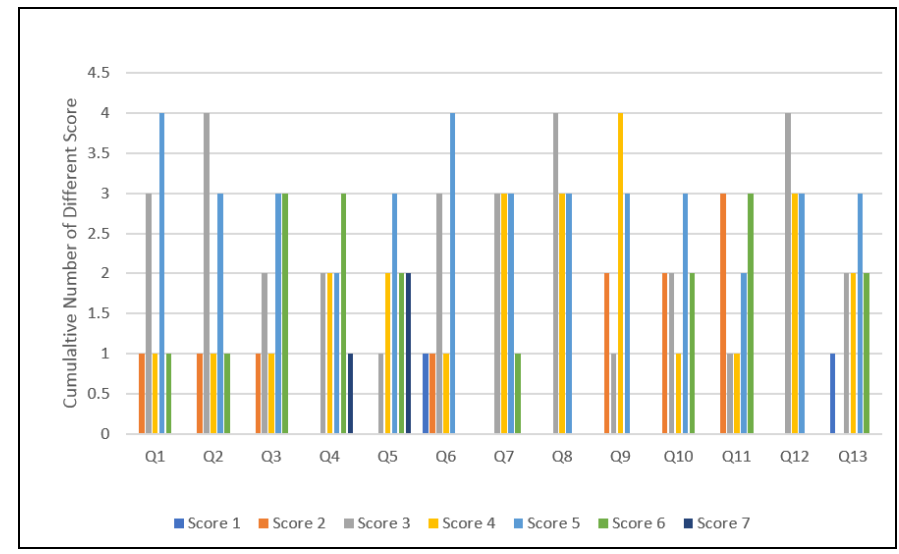

Fig. 5. Cumulative score for each question.

In general, the elderly had responded positively in the survey. It is observed that the bars are slightly sided to the right side of the graph (Fig. 5). The higher mean of Q4 and Q5 shows that the elderly were positive on the arguments that the game has successfully triggered their curiosity and able to keep their attention. The less agreed point is Q6 where they felt that the game was lack of customizable features which can encourage them to engage with the activities. The lower score in Q2 and Q12 shows that the elderly felt that the game is easy and not challenging enough. This concludes that the elderly were interested in more advance gaming design; this is contradicting with the perception that the elderly may have problem coping with the mobile technology and prefer the basic and simple gaming features.

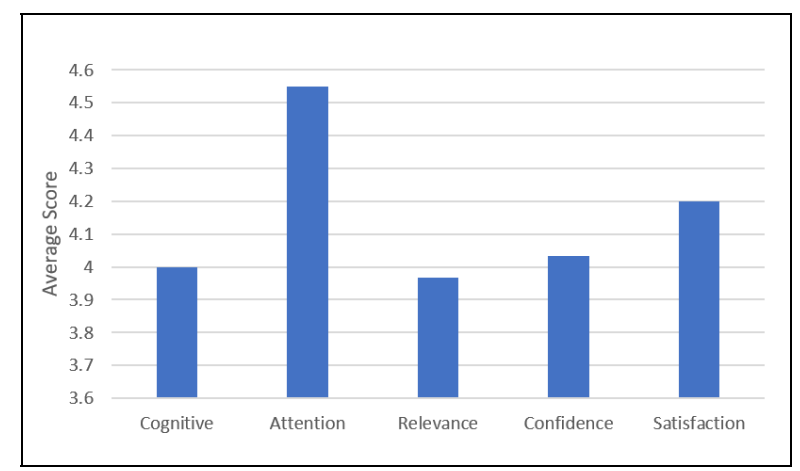

Fig. 6. Average score by ARCS + Cognitive grouping

The average scores grouped by ARCS are shown in Fig. 6. The results show a stronger positive response on the attention and satisfaction grouping. This indicates that the elderly were satisfied and attracted to play the game. Majority think that the task catches their attention (the game will constantly call number which needs the user's attention), provides them curiosity (the different style of the bingo and responsive game has encouraged them to explore the game), needs them to focus on different activities (the user needs to listen to the number while looking at the numbers on multiple boards) and encourages them to be more engaged to the game. The least agreed grouping is relevance where the elderly felt that the 
Bingo game is less relevance to their daily life activities and they were having difficulty to understand and relate the game instructions to complete the game. The feedbacks suggest that the game design should incorporate contents related to the elderly daily activities, for e.g. gardening, cooking, exercises.

TABLE III. CORRELATION ANALYSIS OF ARCS

\begin{tabular}{|lrrrrr|}
\hline \multicolumn{7}{c}{ Cognitive } & Attention & Relevance & Confidence & Satisfaction \\
\hline Cognitive & 1.0000 & & & & \\
Attention & $\mathbf{0 . 7 5 8 3}$ & 1.0000 & & & \\
Relevance & -0.1131 & -0.2048 & 1.0000 & & \\
Confidence & 0.0446 & -0.0682 & $\mathbf{0 . 6 4 1 1}$ & 1.0000 & \\
Satisfaction & 0.2641 & -0.0755 & 0.4522 & $\mathbf{0 . 6 3 3 0}$ & 1.0000 \\
\hline
\end{tabular}

When analyzing the Pearson correlation coefficient between the different ARCS and cognitive groupings, it is observed that a significant correlation of attention and cognitive is found at $r=.7583, \mathrm{p}<.01$. Besides that, strong correlations are also found between relevance and confidence at $r=.6411, \mathrm{p}<.05$, and between confidence and satisfaction at $r=.6330, \mathrm{p}<.05$ as listed in TABLE III. These findings support the hypothesis of 1) The activities that required more mental efforts and have higher difficulty, successfully catches the elderly attention and draw their curiosity to complete the task, 2) A game with clearer guidance and instructions on how to perform the task and able to relate to the elderly real life activities can increase the elderly's confidence in playing the game, 3) Elderly who have confidence in performing and completing the challenging tasks have a pleasant and higher satisfaction on overall user experience.

\section{CONCLUSION}

In conclusion, this project has successfully demonstrated the developing of a customized bundled Bingo mobile game for the elderly users. The development of this application was based on the interface design guideline to suit the needs of the elderly. Generally, majority of the participants have shown a positive response to the acceptance of the mobile game. The analysis results have shown that the elderly preferred the gaming content which relate to their daily life activities. The elderly also expressed their interest in the game with increased difficulty as well as more flexibility and customizable options. The clear instruction and guidance on how to play the game is also one of the important factor which will directly impact the elderly's confidence, and hence influence the overall user experience and satisfaction in playing the game.

Based on the findings, this mobile-based Bingo bundled game still has room to improve to better suit the interest of the elderly. One aspect that can be worked on is to engage a bigger tester group of active elderly to further study and analyze the usage behavior.

\section{ACKNOWLEDGMENT}

Thanks to Sunway University, Assoc. Prof. Dr. Wong Siew Fan and Assoc. Prof. Dr. Lee Yun Li for contribution in the earlier discussions.

\section{REFERENCES}

[1] N. Hamizah \& F.H.A. Razak, "Smart Phone Menu Layout for Elderly Users", in Proceedings of the 5th International Conference on Computing and Informatics, ICOCI 2015, pp. 247-253, 2015.

[2] B. Cook and P. Twidle, "Increasing Awareness of Alzheimer's Disease through a Mobile Game," 2016 Int. Conf. Interact. Technol. Games, pp. 55-60, 2016.

[3] Yee et al, "Current Progress in Interaction Design for Seniors", $\mathrm{CHI}$ '10 Workshop on Senior-friendly Technologies: Interaction design for the Elderly', pp. 3-6, 2010.

[4] Duh et al, "Senior-Friendly Technologies: Interaction Design for Senior Users", CHI '10 Extended Abstracts on Human Factors in Computing Systems, pp. 4513-4516, 2010.

[5] A. Holzinger, G. Searle and A. Nischelwitzer, "On Some Aspects of Improving Mobile Applications for the Elderly", International Conference on Universal Access in Human-Computer Interaction, UAHCI 2007, pp. 923-932, 2007.

[6] W. IJsselsteijn, H.H. Nap and Y. de Kort, "Digital Game Design for Elderly Users", in Proceedings of the 2007 conference on Future Play, pp. 17-22, 2007.

[7] K.M. Gerling, F.P. Schulte and M. Masuch, "Designing and Evaluating Digital Games for Frail Elderly Persons", in Proceedings of the 8th international conference on advances in computer entertainment technology, 2011.

[8] B. de Schutter and V.V. Abeele, "Designing meaningful play within the psycho-social context of older adults", in Proceedings of the $3 \mathrm{rd}$ International Conference on Fun and Games, pp. 84-93, 2010.

[9] H.H. Nap, W.A. Ijsselsteijn and Y.A.W. de Kort, "Age Differences in Associations with Digital Gaming", in Digital Games Research Association Conference, 2009.

[10] M. Rautenberg, "Positive Effects of Entertainment Technology on Human Behaviour", IFIP International Federation for Information Processing, pp. 51-58, 2004.

[11] Kobayashi et al, "Elderly User Evaluation of Mobile Touchscreen Interactions", in IFIP Conference on Human-Computer Interaction, pp. 83-99, 2011.

[12] Vasconcelos et al, "Designing Tablet-Based Games for Seniors: the example of CogniPlay, a cognitive gaming platform", in Proceedings of the 4th International Conference on Fun and Games, 2012.

[13] Aison et al. (2002, May). Appeal and Interest of Video Game Use Among the Elderly. [Online]. Available: http://www.booizzy.com/jrmilner/portfolio/harvard/gameselderly.pdf

[14] Loughborough University Institutional Repository. (2014, July). Touchscreen mobile devices and older adults: a usability study. [Online]. Available:https://dspace.lboro.ac.uk/dspace-jspui/bitstream/2134/17187/ 3/Touchscreen.pdf

[15] K.M. Gerling, F.P. Schulte and M. Masuch, "Designing and Evaluating Digital Games for Frail Elderly Persons", in Proceedings of the 8th international conference on advances in computer entertainment technology, 2011.

[16] W.A. Ijsselsteijn, H.H. Nap, and Y.A.W. de Kort, "Digital Game Design for Elderly Users", in Proceedings of the 2007 conference on Future Play, pp. 17-22, 2007.

[17] R.N.S. de Carvalho and L. Ishitani, "Motivational Factors for Mobile Serious Games for Elderly Users", in SBC-Proceedings of SBGames 2012, pp. 19-28, 2012.

[18] J.A. lain and M. Shen. (2015, September). Perception and Misperception. [Online]. Available: http://carnegieendowment.org/files/SecurityPerceptions_finall.pdf

[19] B. de Schutter and V.V. Abeele, "Designing meaningful play within the psycho-social context of older adults", in Proceedings of the 3rd International Conference on Fun and Games, 2010.

[20] Dictionary.com. (2014). Motor Skills. Dictionary.com, LLC. [Online]. Available: http://www.dictionary.com/browse/motor-skills

[21] S.F. Wong, “ARCS questionnaire”, Sunway University, 2016. 